\title{
INCIDÊNCIA DE ALTERAÇÕES DENTÁRIAS EM UM REBANHO BOVINO LEITEIRO
}

\author{
(Incidence of dental lesions in a dairy herd ) \\ OLLHOFF, R.D.'; PEREIRA, I.R.A. ${ }^{2}$; LUZ, N.C. ${ }^{2}$; MACHADO, F.G. ${ }^{3}$ \\ 'Pontifícia Universidade Católica do Paraná, R. José Francisco Dalledone 237, 82200-250, Curitiba, \\ Paraná daniel.ollhoff@pucpr.br; \\ ${ }^{2}$ Graduação em medicina veterinária da PUCPR; \\ ${ }^{3}$ Médico veterinário residente da PUCPR.
}

RESUMO - O presente experimento objetivou levantar a incidência de alterações dentárias em bovinos leiteiros criados intensivamente. Realizou-se o exame clínico da cavidade oral de 64 vacas em lactação mantidas em sistema de "free-stall" com duas ordenhas diárias, sendo o alimento oferecido em comedouro cimentado. Foram examinadas 16 vacas da raça Pardo-Suíça e 48 vacas da raça Holstein Friesian com idade média de 48,1 meses (21 a 108 meses de idade). Em 38 vacas $(59,4 \%)$ utilizou-se a grosa dental com cabeça móvel para raspagem dos dentes, seguido de antiséptico bucal (15 ml de solução de violeta de genciana a 1\% p.o.). Em 26 animais (40,6\%) não foi necessária nenhuma intervenção. As alterações mais comuns encontradas foram desgastes da superfície oclusal de molares e pré-molares em escada com formação de pontas excessivamente proeminentes e afiadas em 37 animais (57,8\%). Os desgastes ocorreram em $28,1 \%$ dos pré-molares superiores direitos e esquerdos, em 4,7\% dos pré-molares inferiores direitos e em $6,3 \%$ dos prémolares inferiores esquerdos. Os molares apresentavam o mesmo desgaste excessivo em 20,3\% dos superiores direitos e $18,8 \%$ dos superiores esquerdos. Os molares inferiores direitos e esquerdos participaram respectivamente com 9,4\% e 7,8 \%. Foram ainda observados 4 casos de gengivite em incisivos (6,3\%), 4 casos de fratura de incisivos (6,3\%), 3 casos de desgaste em meia lua de incisivos na face oclusal (4,7\%), 3 casos de desgaste de incisivos em face labial $(4,7 \%), 2$ casos de persistência dos incisivos decíduos (3,2\%), um caso de úlcera em mucosa lingual e bucal $(1,6 \%)$, um caso de desgaste excessivo de incisivo com exposição de polpa dentária e fratura $(1,6 \%)$ e um caso de hipoplasia dentária $(1,6 \%)$. Conclui-se que o exame da cavidade oral dos bovinos leiteiros constitui um instrumento importante para a promoção da saúde e produtividade do rebanho.

Palavras-chave: dente, bovino, incidência, alterações.

ABSTRACT - The oral cavity of 64 milk cows was examined. They were confined in a free-stall, milked twice daily and fed with TMR- total mixed ration. A total of 16 Brown Swiss and 48 Holstein Friesian cattle with medium age of 48,1 months (from 28 to 108 months of age) were examined. The teeth of 38 cows $(59,4 \%)$ were rasped and rinsed afterwards with $15 \mathrm{ml}$ of gentian-violet at $1 \%$. It has been found that 26 cows $(40,6 \%)$ displayed no detectable alterations. The most commonly found alterations were premolar and molar oclusal surface wear, forming sharp edges in 37 animals $(57,8 \%)$. The wear occurred in $28,1 \%$ of the cases on the left and right superior premolar teeth, in $4,7 \%$ of the cases on the right inferior premolars and $6,3 \%$ on the left inferior premolars. A total of $20,3 \%$ of the right superior molars, $18,8 \%$ of the left superior, $9,4 \%$ of the right inferior and $7,8 \%$ of the left inferior molars showed sharp edges. Furthermore, it has been observed $4(6,3 \%)$ cases of gingivitis, $4(6,3 \%)$ cases of fracture of the incisors, $3(4,7 \%)$ cases of half-moon shaped occlusive wear, $3(4,7 \%)$ cases of labial wear of the incisors, $2(3,2 \%)$ cases of deciduous teeth persistence, $1(1,6 \%)$ case of oral mucosa ulceration, $1(1,6 \%)$ case of excessive wear with pulpa exposure and fracture and $1(1,6 \%)$ case of dental hypoplasia. It can be concluded that oral cavity examination is a valuable instrument to promote health and productivity in dairy herds.

Key-words: teeth, cattle, incidence, lesions. 


\section{Introdução}

A cavidade oral e os dentes fazem parte do aparelho digestório (SISSON 1986). Apesar disto, na clínica de bovinos, o exame desta região é negligenciado pelo esforço físico e material que este exame exige e o risco em potencial de ferimentos do examinador por esmagamento ou cortes provocados principalmente pela dentição.

O bovino apresenta como característica uma apreensão do alimento predominantemente com a língua e partículas pequenas também com os lábios. A mastigação ocorre com movimentos de moagem, estes sendo mais evidentes na ruminação do que na apreensão do alimento inicial (DIRKSEN 1990). O bovino apresenta na idade adulta a seguinte fórmula dentária (ZIETZSCHMANN et al. 1985):

$$
\frac{0 I 3 P 3 M}{4 I 3 P 3 M}
$$

O exame da dentição bovina mereceu importância na literatura consultada principalmente visando estabelecer-se uma identificação etária precisa (BROOKES e HODGES 1979; GRAHAM e PRICE 1982; WIENER e FORSTER 1982) ou na ocorrência de surtos de intoxicação pelo flúor (KROOK et al. 1983; ECKERLIN et al. 1986; MAYLIN et al. 1987; ARAYA et al. 1990; BOTHA et al. 1993). Esporadicamente foram realizados relatos por ROGERS e POOLE (1987) e ROGERS e COMERFORD (1992) de desgastes dos incisivos em vacas alimentadas com silagem. Somente mais recentemente a questão do desgaste dos incisivos mereceu uma investigação no Brasil por parte de EURIDES et al. (2000), que investigaram o tipo e profundidade do desgaste dos incisivos em vacas leiteiras em Minas Gerais.

O presente trabalho visa relatar a incidência de alterações dentárias, tanto de incisivos quanto de pré-molares e molares, de um rebanho bovino leiteiro mantido em confinamento em sistema de "free-stall" e as medidas terapêuticas e profiláticas adotadas.

\section{Material e Método}

Para o levantamento foi utilizada a totalidade das 64 vacas em lactação no momento do exame (abril de 2005). O rebanho era oriundo de uma propriedade localizada no município de Fazenda Rio Grande no estado do Paraná, sul do Brasil. As vacas eram mantidas em sistema de "free-stall" com duas ordenhas diárias e com o alimento oferecido na forma de ração total (volumoso misturado ao concentrado acrescido aos sais minerais) oferecido em comedouro cimentado em paralelo ao canzil. $\mathrm{O}$ alimento era oferecido após a ordenha. $O$ rebanho era composto por 16 vacas Pardo-Suiças e 48 vacas Holandesas com idade média de 48,1 meses (21 a 108 meses de idade). Os animais foram contidos através do canzil individualmente, utilizando-se um abre-boca de Schulze. A língua era segura com auxílio de uma toalha e tracionada pela comissura lateral da boca trocando-se os lados respectivamente para o exame dos molares e pré-molares direitos ou esquerdos. Uma lanterna iluminava e facilitava a inspeção da cavidade. A palpação manual era realizada somente em casos de suspeita de dentes soltos. Os achados eram anotados individualmente utilizando-se as denominações Pré-molares $(1,2,3)$ superiores ou inferiores, direitos ou esquerdos (ex.: PM, SE = Pré-molar 1 superior esquerdo); Molares $(1,2,3)$ superiores ou inferiores, direitos ou esquerdos e Incisivos $(1,2,3,4)$ direitos ou esquerdos. Para correções utilizou-se uma grosa dental com cabeça móvel para raspagem dos dentes, seguido de anti-séptico bucal (15 $\mathrm{ml}$ de solução de violeta de genciana a $1 \%$ p.o.). Os resultados foram compilados através de estatística descritiva simples (freqüências relativas).

\section{Resultados}

De 64 animais examinados, 26 animais foram considerados sadios $(40,6 \%)$ não sendo necessária nenhuma intervenção. Em 38 vacas $(59,4 \%)$ utilizou-se a grosa dental com cabeça móvel para raspagem dos dentes, seguido de anti-séptico bucal (15 ml de solução de violeta de genciana a $1 \%$ p.o.). As alterações mais comuns encontradas foram desgastes da 
Incidência de alterações dentárias em um rebanho bovino leiteiro

superfície oclusal de molares e pré-molares em escada com formação de pontas excessivamente proeminentes e afiadas em 37 animais (57,8\%). A distribuição das alterações dentárias encontra-se se na TABELA 1. Alterações nos incisivos foram observados em $30 \%$ das vacas, sendo que as alterações mais notórias encontram-se nas FIGURAS 1 a 4.

TABELA 1 - TIPO DE ALTERAÇÕES DENTÁRIAS E SUA RESPECTIVA LOCALIZAÇÃO EM BOVINOS LEITEIROS ( $\mathrm{N}=64$ ) EM SISTEMA DE CRIAÇÃO INTENSIVA DO TIPO ESTABULAÇÃO LIVRE NO MUNICÍPIO DE FAZENDA RIO GRANDE - PR (2005).

\begin{tabular}{|c|c|c|c|c|c|c|c|c|c|}
\hline & \multirow[b]{3}{*}{ Incisivos } & \multicolumn{4}{|c|}{ Esquerdo } & \multicolumn{4}{|c|}{ Direito } \\
\hline & & \multicolumn{2}{|c|}{ Superior } & \multicolumn{2}{|c|}{ Inferior } & \multicolumn{2}{|c|}{ Superior } & \multicolumn{2}{|c|}{ Inferior } \\
\hline & & PM & M & PM & $\mathrm{M}$ & PM & M & PM & $\mathrm{M}$ \\
\hline $\begin{array}{l}\text { Desgaste oclusal em escada com } \\
\text { pontas afiadas (\%) }\end{array}$ & & 28,1 & 18,8 & 6,3 & 7,8 & 28,1 & 20,3 & 4,7 & 9,4 \\
\hline Desgaste face oclusal em meia lua (\%) & 4,7 & & & & & & & & \\
\hline Desgaste em face labial (\%) & 4,7 & & & & & & & & \\
\hline Fratura (\%) & 6,3 & & & & & & & & \\
\hline Exposição de polpa e fratura (\%) & 1,6 & & & & & & & & \\
\hline Persistência (\%) & 3,2 & & & & & & & & \\
\hline Hipoplasia dentária (\%) & 1,6 & & & & & & & & \\
\hline Gengivite (\%) & 6,3 & & & & & & & & \\
\hline Inserção oblíqua (\%) & 1,6 & & & & & & & & \\
\hline Total (\%) & 30 & & & & & & & 14 & \\
\hline
\end{tabular}

$\mathrm{PM}=$ dente pré-molar; $\mathrm{M}=$ dente molar.

\section{Discussão e Conclusões}

Quando trabalha-se com odontologia buiátrica, o exame dental individualizada, dente por dente, preconizado por ROBINSON e GORREL (1995) para cães e gatos não pode ser utilizado sem adaptações. Os incisivos ainda podem ser facilmente examinados por encontrarem-se em local de fácil acesso dentro da cavidade oral e apresentarem-se normalmente limpos. No entanto quando se examinam os pré-molares e molares, principalmente os inferiores o exame é rotineiramente dificultado pela quantidade de ingesta depositada ao redor dos dentes. A abundante salivação, estimulada pela manipulação do examinador faz com que estes dentes rapidamente encontrem-se imersos em saliva, impedindo muitas vezes um detalhamento do exame. Estas dificuldades podem ser responsabilizadas em parte pelos números mais baixos de incidência de alterações encontradas nos pré-molares e molares inferiores neste estudo. Nenhum dos autores da literatura consultada e listada acima menciona o exame dos pré-molares e molares "in vivo", sendo que somente KROOK et al. (1983) descreve as alterações de fluorose nestes dentes na necropsia. Como a intervenção nos pré-molares e molares restringe-se normalmente a uma apara de arestas pontiagudas e afiadas através da grosa, sugere-se que sejam tratados no exame buiátrico da cavidade oral em conjuntos (os três pré-molares superiores esquerdos, os três molares inferiores direitos etc.). Esta medida acelera o processo de exame importante quando se examinam rebanhos inteiros. À semelhança de EURIDES et al. (2000) encontrou-se desgastes da face oclusal dos incisivos em formato de meia lua (FIGURA 1), denominados por estes autores de desgastes irregulares. Afora um animal com a polpa exposta por desgaste excessivo do incisivo (FIGURA 2) não se encontrou mais nenhuma lesão endodôntico e nem de impacção infundibular, descrito por DUBIELZIG (1990) como sendo de importância no ruminante. Uma explicação para isto pode ser o manejo mineral otimizado dos animais e a baixa idade média do rebanho. O desgaste dos incisivos em sua face labial em 3 animais pode ser decorrente de maneirismos ou etopatias, pois os três animais foram relatados pelos tratadores como sendo animais que possuem o hábito de roer as estruturas metálicas (canos) do canzil. A 
hipoplasia dentária, atingindo tanto dentina como esmalte como demonstra a FIGURA 4 julgamos ser, como ocorreu em ambos os incisivos centrais $\left(\mathrm{I}_{1} \mathrm{E}\right.$ e $\left.\mathrm{I}_{1} \mathrm{D}\right)$ uma hipoplasia de Turner, isto é, uma forma de amelogênese imperfeita caracterizada pela formação incompleta do esmalte dentário. No homem, a hipoplasia do esmalte pode ser decorrente de um traumatismo, deficiência de vitaminas $A, C$ ou $D$, infecções virais (sarampo, escarlatina) prematuridade e infecções locais entre outros (RIBAS e CZLUSNIAK 2004). Pelo histórico obtido da vaca não foi possível estabelecer a possível origem deste achado. Desconhecemos a descrição desta alteração dentária em bovinos.

FIGURA 1 - INCISIVOS PERMANENTES COM DESGASTE EM MEIA LUA. OBSERVA-SE PRESENÇA DE ALIMENTO ENTRE OS DENTES.

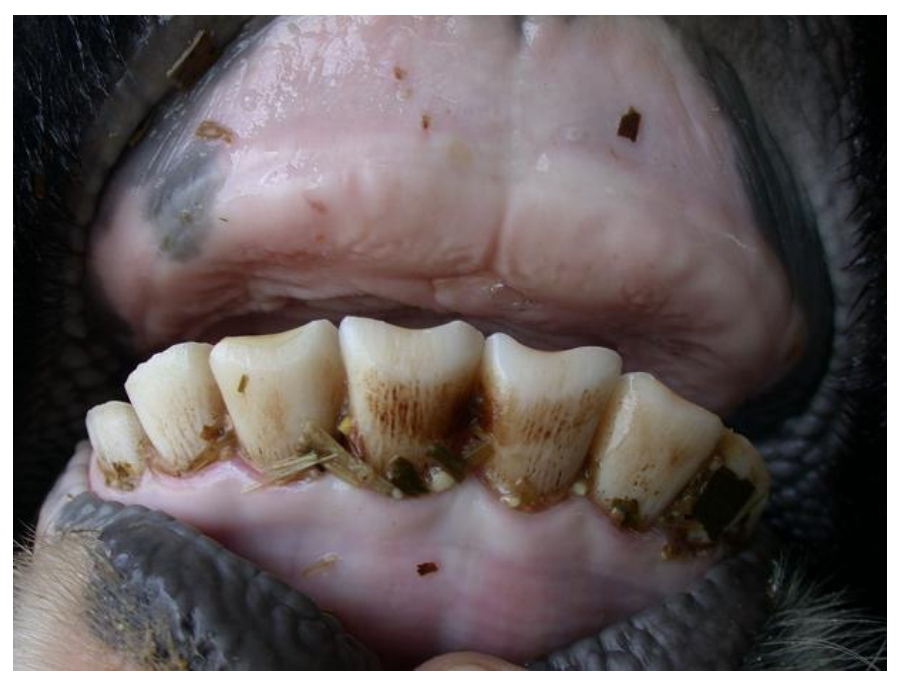

FIGURA 2 - DESGASTE SEVERO DE INCISIVO MEDIAL ESQUERDO COM EXPOSIÇÃO DA POLPA E FRATURA.

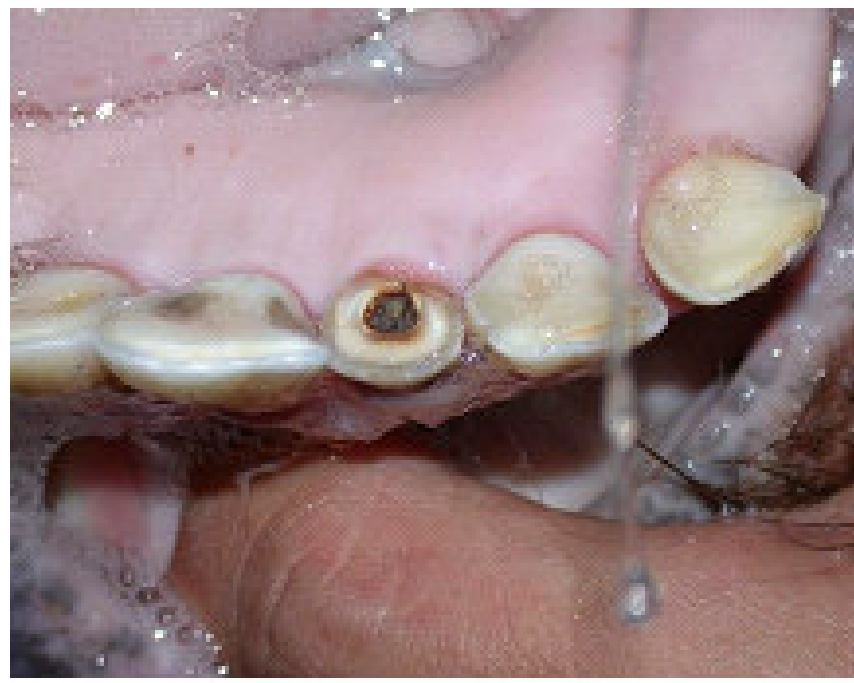


FIGURA 3 - INCISIVO EXTERNO ESQUERDO EM POSIÇÃO OBLÍQUA.

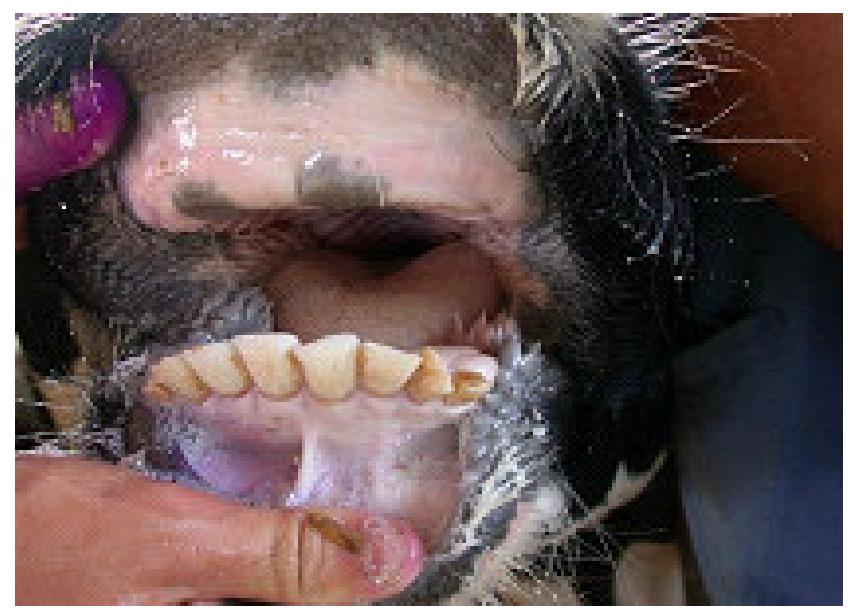

FIGURA 4 - HIPOPLASIA DENTÁRIA DE AMBOS OS INCISIVOS CENTRAIS. OBSERVA-SE INCISIVO DECÍDUO QUE AINDA NÃO CAIU, APESAR DA ERUPÇÃO DO PERMANENTE.

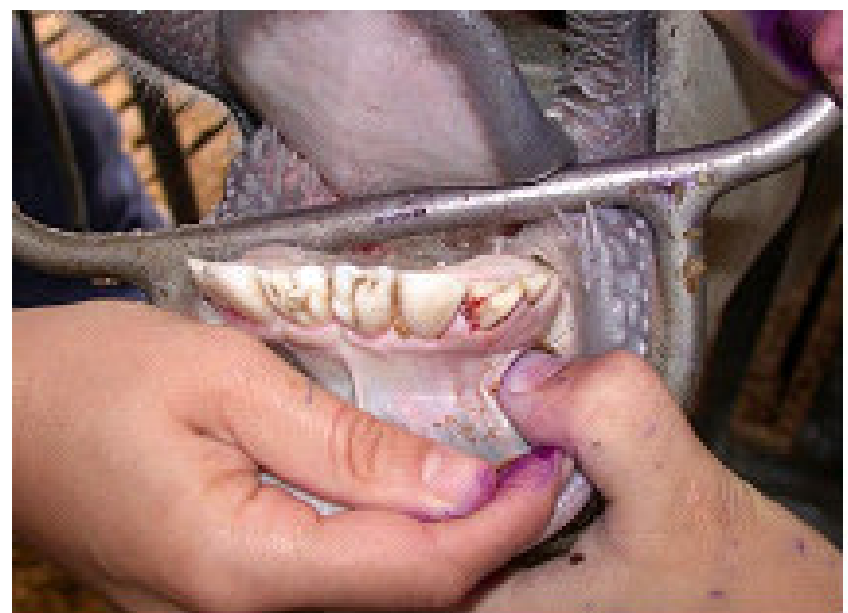

Para determinar se a acidez da silagem poderia ter contribuído no desgaste irregular dos dentes como sugerem ROGERS e POOLE (1987) e ROGERS e COMERFORD (1992) ou se é apenas a conseqüência de formas de mastigação ligeiramente alteradas haveria a necessidade de se aprofundar os estudos.

Conclui-se que: o exame rotineiro da cavidade oral com especial atenção aos dentes, constitui adicionalmente um serviço profilático que o médico veterinário buiatra poderá oferecer, para promover a saúde nos rebanhos e otimizar consequentemente a produtividade; a raspagem dos dentes, seguida da aplicação de solução de violeta de genciana a $1 \%$, constituiu-se em medida terapêutica.

\section{REFERÊNCIAS}

ARAYA, O.; WITTWER, F.; VILLA, A.; DUCOM, C. Bovine fluorosis following volcanic activity in the southern Andes. Veterinary Record, v.126, p.641642, 1990.

BOTHA C.J.; NAUDÉ, T.W.; MINNAAR, P.P; VAN AMSTEL, S.R.; VAN RENSBURG, S.D.J. Two outbreaks of fluorosis in cattle and sheep. Journal of the South African Veterinary Association, v.64, n.4, p.165-168, 1993. 
BROOKES, A.J.; HODGES, J. Breed. Nutritional and heterotic effects on age of teeth emergence in cattle. Journal of Agricultural Science, v.93, p.681-685, 1979.

DIRKSEN, G. Verdauungsapparat. In: ROSENBERGER, G. Die klinische Untersuchung des Rindes. 3 ed. Berlin und Hamburg: Verlag Paul Parey, 1990, p.288-400.

DUBIELZIG, R.R. Patologia dental. In: Thomson, R. G. Patologia Veterinária Especial. São Paulo, Editora Manole Ltda., 1990, p.143-153.

ECKERLIN, R.H.; MAYLIN, G.A.; KROOK, L. Milk production of cows fed fluoride contaminated commercial feed. Cornell Veterinarian, Ithaca, v.76, p.403-414, 1986.

EURIDES, D.; SILVA, F.O.C.; SILVA, L.A.F.; FIORAVANTI, M.C.S.; CAMPOS, V.A.; KANAYAMA, C. Y. Desgastes dos dentes incisivos em vacas leiteiras. Revista Científica Rural, v.5, n.2, p.141147, 2000.

GRAHAM, W.C.; PRICE, M.A. Dentition as a measure of physiological age in cows of different breed types. Canadian Journal of Animal Science, Alberta, v.62, p.745-750, 1982.

KROOK, L.; MAYLIN, G.A.; LILLIE, J.H.; WALLACE, R.S. Dental fluorosis in cattle. Cornell Veterinarian, Ithaca, v.73, p.340-362, 1983.

Recebido para publicação: 30/06/2005

Aprovado:

$20 / 09 / 2005$
MAYLIN, G.A.; ECKERLIN, R.H.; KROOK, L. Fluoride intoxication in dairy calves. Cornell Veterinarian, Ithaca, v.77, p.84-98, 1987.

RIBAS, A.O.; CZLUSNIAK, G.D. Anomalias do esmalte dental: etiologia, diagnóstico e tratamento. Publicações da UEPG Ciências Biológicas e da Saúde, v.10, n.1, p.23-36, 2004.

ROBINSON, J.; GORREL, C. Oral examination and radiography. In: CROSSLEYD. A.; PENMANN, S. BSVA Manual of small animal dentistry. $2 \mathrm{ed}$. Gloucestershire, British Small Veterinary Association, p.35-49, 1995.

ROGERS, P.A.M.; COMERFORD, P.J. Erosion of the incisors of silage-fed cattle. Veterinary Record, London, v.130, n.20, p.455-456, 1992.

ROGERS, P.A.M.; POOLE, B.R. Incisor wear in cattle on self-fed silage. Veterinary Record, London, v.120, n.14, p.348, 1987.

SISSON, S. Aparelho digestório. In: GETTY, R. Sisson / Grossmann Anatomia dos Animais domésticos. 5 ed., Rio de Janeiro, Editora Guanabara, p.100-107, 1986.

WIENER, G.; FORSTER, J. Variation in the age at emergence of incisors teeth in cattle of different breeds. Animal Production, Praha, v.35, 367-373, 1982.

ZIETZSCHMANN, O.; ACKERKNECHT, E.; GRAU, H. Ellenberger-Baum Handbuch der vergleichenden Anatomie der Haustiere. 18. ed. Berlin, Heidelberg, New York: Springer Verlag, 1985. 1155p. 